\title{
Effects of Shell-Buckling Knockdown Factors in Large Cylindrical Shells
}

\author{
Glenn A. Hrinda ${ }^{1}$ \\ NASA Langley Research Center, Hampton, Virginia, 23681
}

\begin{abstract}
Shell-buckling knockdown factors (SBKF) have been used in large cylindrical shell structures to account for uncertainty in buckling loads. As the diameter of the cylinder increases, achieving the manufacturing tolerances becomes increasingly more difficult. Knockdown factors account for manufacturing imperfections in the shell geometry by decreasing the allowable buckling load of the cylinder. In this paper, large-diameter ( $33 \mathrm{ft}$ ) cylinders are investigated by using various SBKF's. An investigation that is based on finiteelement analysis (FEA) is used to develop design sensitivity relationships. Different manufacturing imperfections are modeled into a perfect cylinder to investigate the effects of these imperfections on buckling. The analysis results may be applicable to large- diameter rockets, cylindrical tower structures, bulk storage tanks, and silos.
\end{abstract}

\section{Nomenclature}

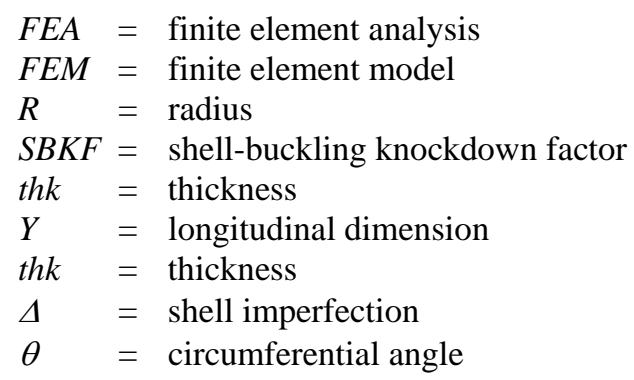

\section{Introduction}

$\mathrm{T}$ HIN-walled cylindrical shells are used in many engineering applications. Their shape and load-carrying capability makes them well-suited for aerospace and civil structures. Additionally, these cylinders are designed with minimum weight and maximum resistance to various load conditions. Aerospace structures (Fig. 1) rely on optimization to minimize weight; similarly, civil engineering structures (Fig. 2) are designed with minimum material to reduce costs.

Unfortunately, the demand to design thinner cylindrical shells and support maximum design loads makes these shells more prone to buckling failure. Further, variations in a cylinder's manufactured dimensions from the design geometry greatly affect determination of the critical buckling load. Classical small deflection theory is not sufficiently accurate when applied to thinwalled cylinders. ${ }^{1}$ The theory

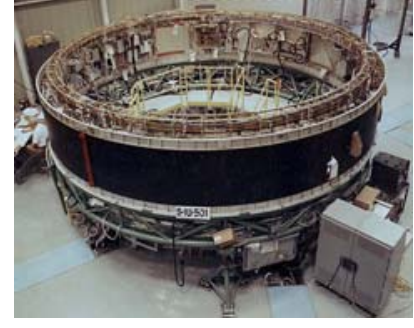

a) Saturn V Instrument Unit

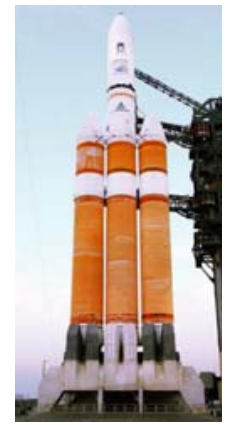

b) Ariane $\mathrm{V}$

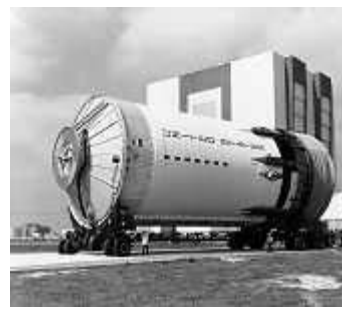

c) Saturn V S-II stage

Figure 1. Cylindrical aerospace structures.

\footnotetext{
${ }^{1}$ Senior Research Engineer, NASA Langley Research Center, Hampton, VA.
} 
typically over predicts the buckling strength when compared with experimental results; this tendency can result in a shell that is too thin. Early aircraft designers discovered this behavior in thin-walled curved panels that were made from sheet metal. They found that the panels buckled at load levels that were significantly lower than those predicted by classical theory. ${ }^{2}$ The reduced buckling levels were caused by geometric imperfections, such as manufactured surface defects, shell-thickness variations, and a difficulty in applying perfect loads to prevent eccentricities.

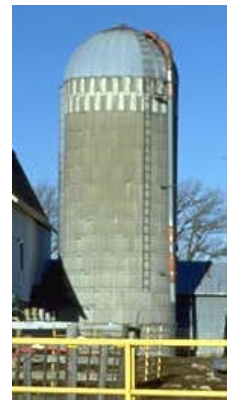

a) Grain

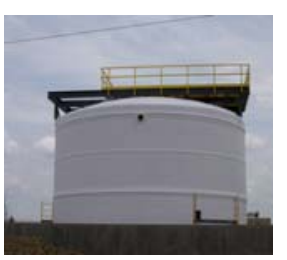

b) Bulk storage tank

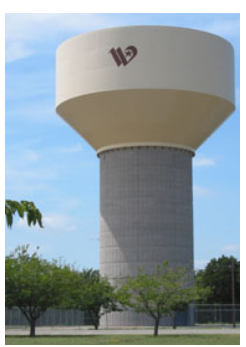

c) Elevated water tank

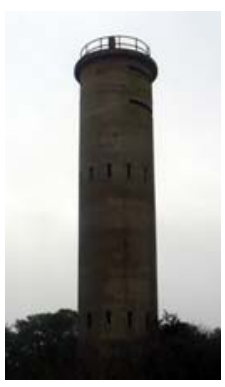

d) Observation tower

Figure 2. Examples of large cylindrical civil engineering structures.

Cylindrical shell designers usually make use of well-known solutions for thin- walled cylinders and modify them with knockdown factors. The knockdown factors are necessary to account for uncertainties in the "as-built" structure. Buckling analysis methods and recommended knockdown factors can be found in reference 3.

Material imperfections may also exist from residual stresses that result from construction techniques and variations in material properties. ${ }^{4}$ Consequently, these imperfections can affect the uncertainty in the design and drive the appropriate knockdown factor to be used in the analysis.

Better results have been demonstrated by using a large deflection theory based on known geometric imperfections. In some designs, attention to construction techniques can reduce the discrepancies that occur between the engineered design and the "as-built" structures. Design standards that address global and local imperfection tolerances may be used which can help in predicting an accurate buckling load. Tolerances that can be used in describing the shape of cylindrical shells are given in reference 5.

Manufacturing imperfections may appear randomly in shell structures; however, they are more likely to occur at connections and joints. ${ }^{6}$ This tendency can be observed in the Ariane III interstage imperfection measurements (Fig. 3 ). The structure is constructed by using eight panels, which are joined with overlapping seams. The plot in Fig. 3 gives a three-dimensional representation of the manufactured shell imperfections that occur along the length of the cylindrical structure. ${ }^{7}$

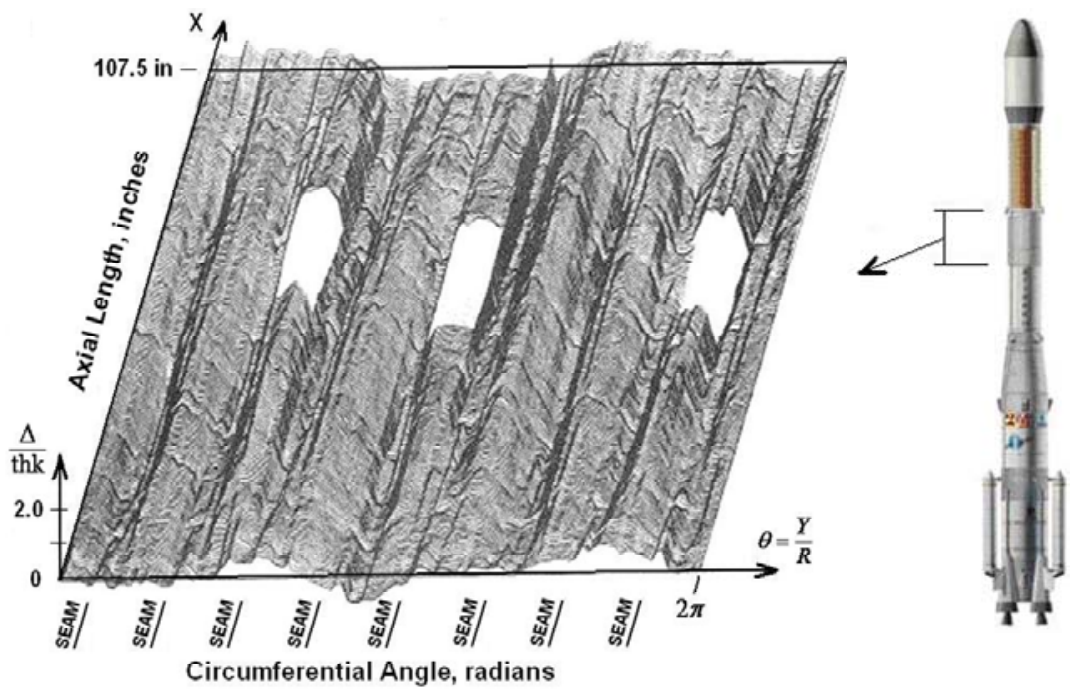

Figure 3. Measured imperfections in Ariane interstage. 


\section{Objectives}

The purpose of this investigation is to determine the correlation between manufacturing imperfections and the SBKF that are used in the design of thin-shelled cylinders. This goal is accomplished by modeling these imperfections into finite-element models (FEM's). The loads are idealized as perfectly applied with no variations allowed. In addition, the material properties are perfectly isotropic with no thermal influences. As a thin-shelled cylinder is axially loaded, the structure buckles, depending on the severity of the imperfections in the shell. If the design does not allow for imperfection, a higher buckling load may be predicted, which can result in an undersized cylinder. Conversely, if a less-effective SBKF is used, then the cylinder may be overdesigned and heavy. Furthermore, the use of the appropriate building tolerances that reflect the SBKF used in the cylinder analysis can help to achieve an adequate design.

\section{Analysis methodology}

The effects of manufacturing imperfections on the design of thin-shelled cylinders were investigated by using a finite-element approach. Large, thin cylinders, each with a diameter of $33 \mathrm{ft}$, were modeled with imperfections that reduced their load-carrying capacity. Each cylinder model was placed under a uniform axial load to cause buckling. Three different cylindrical imperfections were modeled (see Fig. 4) and analyzed by using NASTRAN's eigenvalue buckling solution.

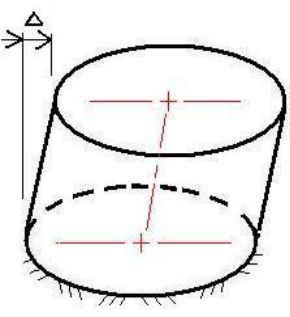

a) Skewed

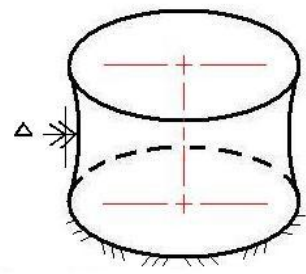

b) Hourglass

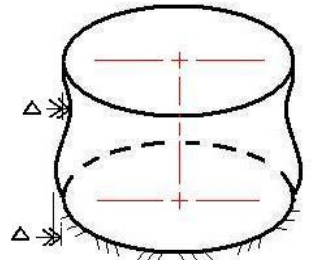

c) Wavy

Figure 4. Imperfection models.

For each imperfection type, the magnitude of the defect was gradually increased. This required the generation of a new FEM for each change to the geometry. A buckling analysis was performed on each FEM. The results were plotted to show the sensitivity of the defect to the buckling load, which is the product of the lowest positive eigenvalue and the total applied load. The expected outcome was that the buckling load would decrease as the severity of the defect increased; likewise, the SBKF was expected to decrease as the degree of defect increased.

The FEM that was used for all of the analyses is based on the model that produced the buckled shape of a perfect cylinder shown in Fig 5. The diameter of the cylinder was 396 inches measured to the center thickness of the elements, and the height was held to 145 inches. These dimensions were selected based on work that is being performed on a stage separation ring for a new rocket concept that has applicability to other large cylindrical structures. ${ }^{8}$ The thickness of the models was 1 inch; standard aluminum material properties were used. The bottom of each cylinder was pin constrained and the upper, open end of each cylinder was left free. A uniform axially compressive load was applied around the upper perimeter of the upper end of each cylinder. Each model was constructed with 207 elements around the circumference and 24 elements along the height. This mesh density was determined sufficient to capture the first buckling mode after investigating models with higher mesh densities. These refined models produced similar buckling results to the model in Fig 5. 


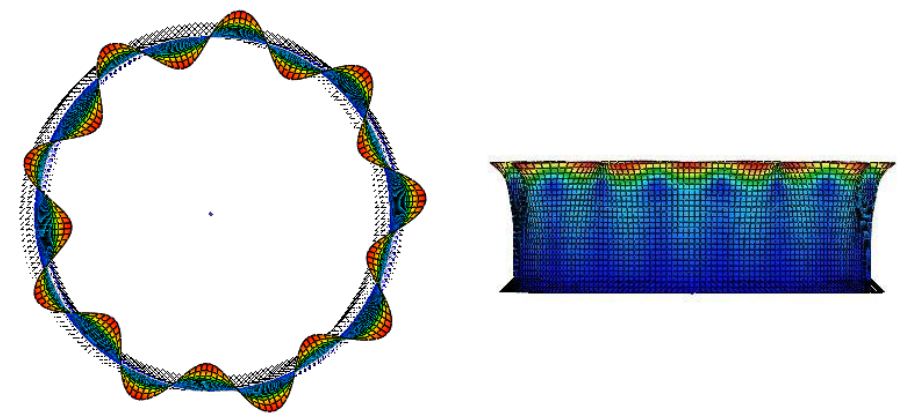

Figure 5. Perfect-cylinder buckled shape.

\section{A. Skewed imperfection}

The first imperfection that was investigated is a skewed, thin-walled cylinder. The defect in the geometry is defined in Fig. 6 as the lateral change in the upper end of the cylinder along one axis. Here, the lateral dimension is governed by the location of the center of the upper diameter as the cylinder is skewed. The plane that is formed at the top end of the cylinder remains parallel to the bottom end of the cylinder. The height of the cylinder was held at 145 inches.
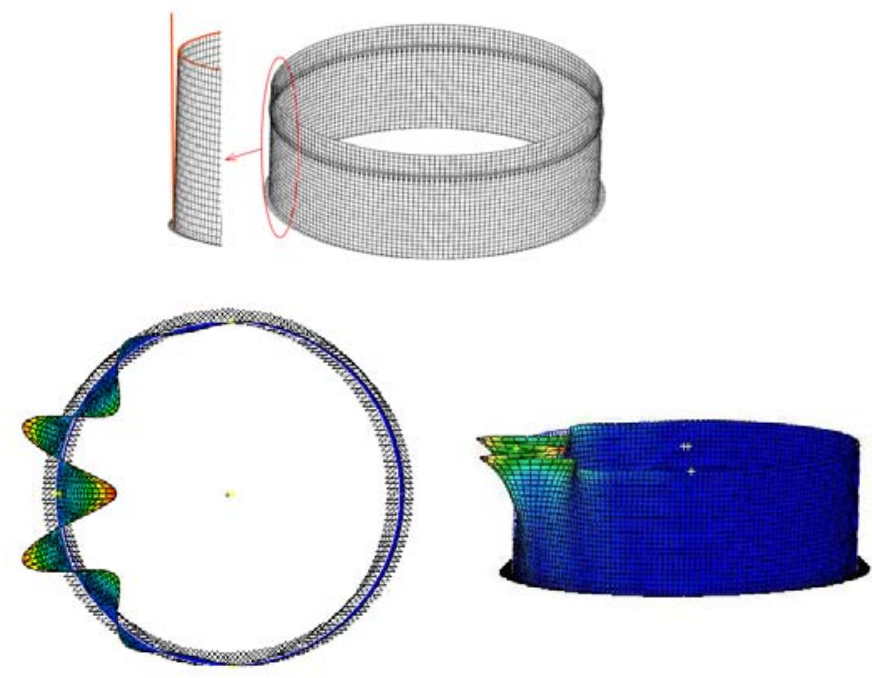

Figure 6. Skewed-model buckled shape.

\section{B. Hourglass imperfection}

The next cylinder imperfection resembles an hourglass shape. The center of the sidewall is forced inward to create the defect. Its curvature from the bottom to the top of the cylinder follows a circular arc. The initial defect starts at $1 / 2$ inch and was increased, as previously mentioned, by $1 / 2$ inch increments. The upper and lower ends of the cylinder are located along the same vertical axis and both ends remain parallel to each other. The height of the cylinder was held at 145 inches. The FEM is shown in Fig. 7. 


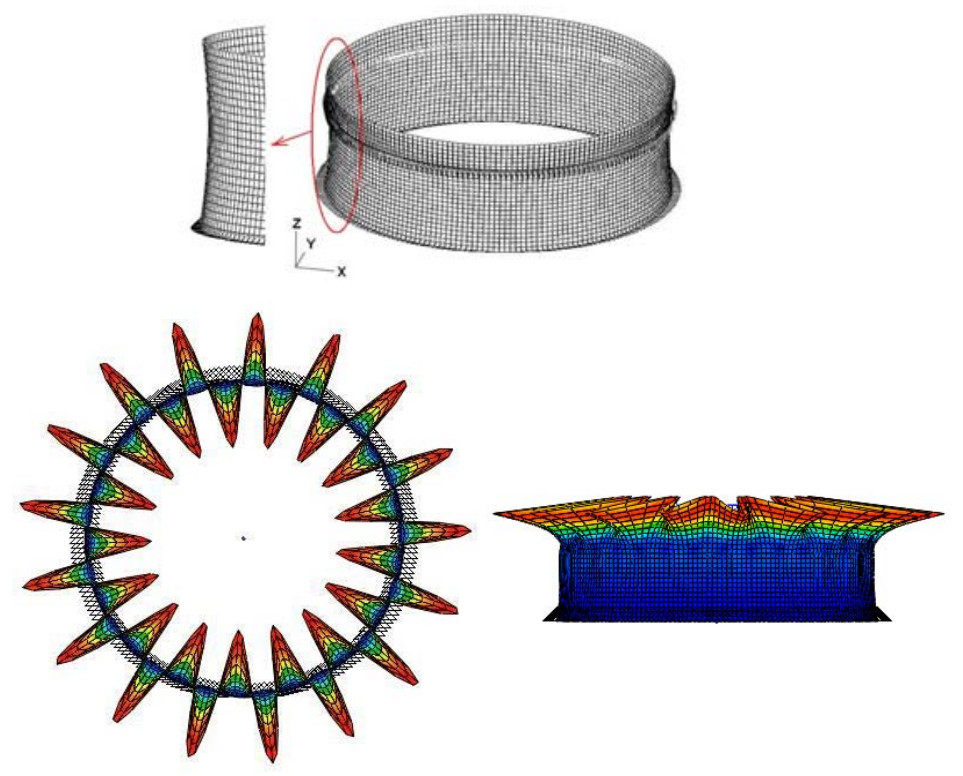

Figure 7. FEM of hourglass buckled shape.

\section{Wavy imperfection}

The third imperfection that was modeled is the wavy type. The bottom half of the sidewall is curved inward, and the upper half is curved outward. Both defects are controlled by the half points along their circular curves. The magnitudes of the defects are equal, but in opposite directions, as can be seen in Fig. 8. The magnitude of the imperfections is changed at 1 inch intervals with a new FEM created for each change. The bottom and top diameters do not change in the lateral directions and remain parallel at 145 inches.
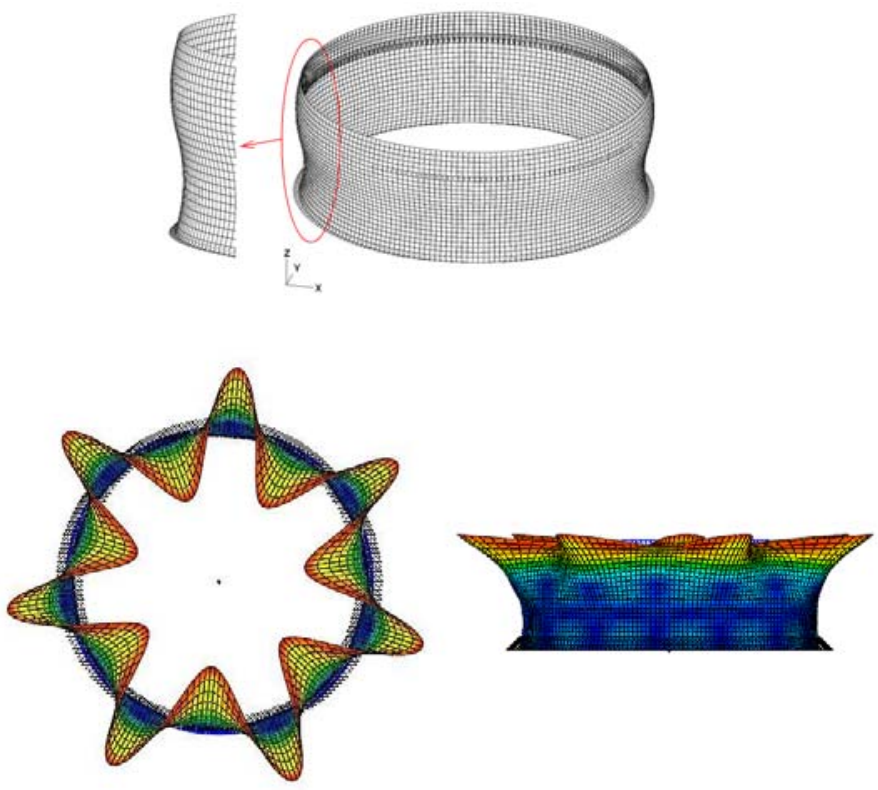

Figure 8. FEM of wavy buckled shape. 


\section{Hyperbolic model}

The hyperbolic model (Fig. 9) was created based on unexpected results obtained from the hourglass imperfection model. The determined buckling load for the hourglass shape, which has sidewalls that curve inward, was slightly greater than that of the perfect cylinder. Based on this result, imperfection models with hyperbolically curved sidewalls were also examined. The circular shape used in the hourglass model was replaced with sides constructed with these hyperbolic functions. The magnitude of the hyperbolic inward apex was varied to demonstrate its effect on the ability of the shell to support a vertical load.
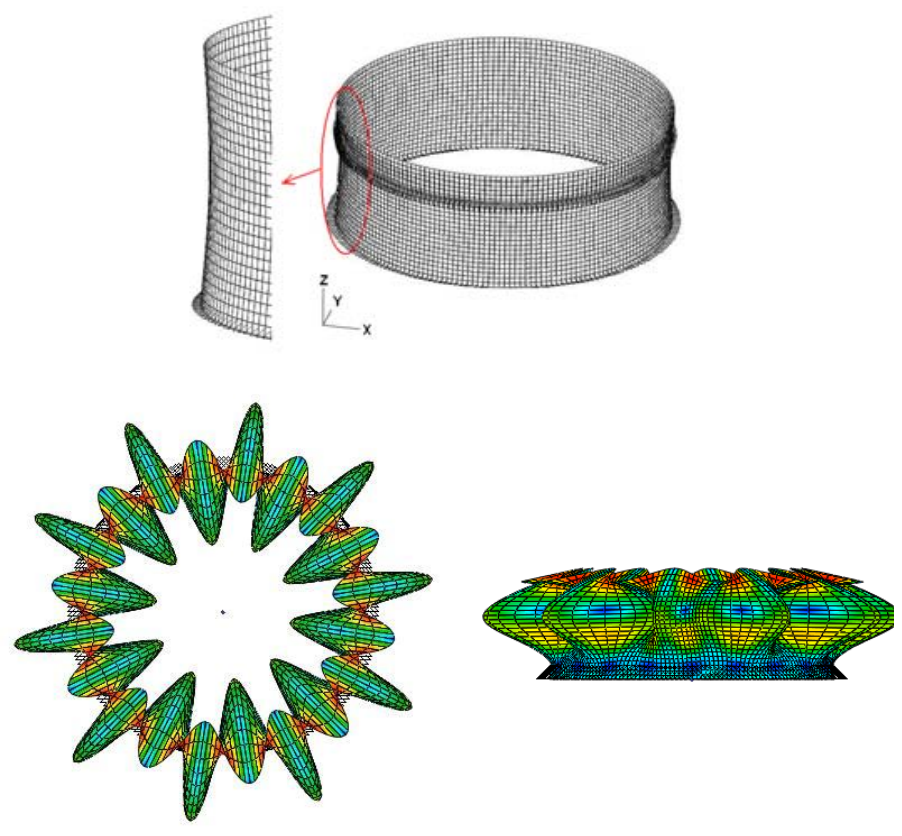

Figure 9. FEM for hyperbolic fem buckled shape.

\section{Results}

The buckling results obtained for the five cylindrical types are plotted in Fig. 10; the numerical values are given in Table 1. In the plot, the vertical axis shows the buckling load at different degrees of imperfection for each of the five shell types. The right axis gives the equivalent SBKF that is necessary to reduce the axial load on the shell structure. The plot permits a comparison of the perfect cylinder results with those for cylinders with various imperfections.

The cylinder with the wavy imperfection shows the greatest decrease in buckling load. In Fig. 10, for a 1 inch imperfection, the buckling load is $32,770 \mathrm{lb}$. This is a reduction in buckling load of more than 50 percent over that of the perfect cylinder. At 2 inches of imperfection, the axial load that the cylinder can support is further reduced to $18,128 \mathrm{lb}$, which is about one-fourth that of the perfect cylinder.

The skewed cylinder has less reduction in buckling load as the degree of imperfection increases. Figure 10 shows that for a 1 inch imperfection, the buckling load for the skewed cylinder decreases to 73,800 lb. The buckling load continues to decrease to $67,095 \mathrm{lb}$ with a 2 inch imperfection. The buckling load for the skewed cylinder is $60,551 \mathrm{lb}$ at 3 inches of imperfection.

The cylinder with the hourglass shape displayed an unexpected increase in buckling load. Figure 10 shows that for a 1 inch imperfection, the load required to buckle the cylinder rises to 89,812 lb, which is an increase of 13 percent over that of the perfect cylinder. At 2 inches, the buckling load drops below that of the perfect cylinder (76,136 lb). This trend continues (see Fig. 10) with a decreased buckling load of 64,513 lb at 3 inches. 
Load vs Imperfection

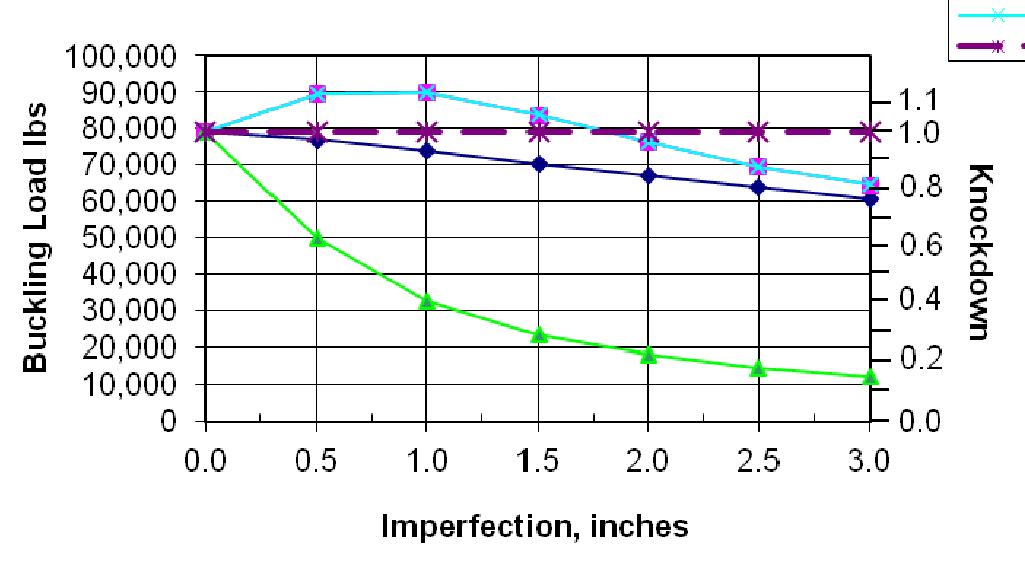

Figure 10. Load versus imperfection results.

Table 1. Load (lbs) versus imperfection (inch) results.

\begin{tabular}{|c|c|c|c|c|c|}
\hline Imperfection & Perfect & Skew & Hourglass & Wavy & Hyperbolic \\
\hline 0.0 & 79250 & 79250 & 79250 & 79250 & 79250 \\
\hline 0.5 & 79250 & 76911 & 89536 & 50068 & 89526 \\
\hline 1.0 & 79250 & 73800 & 89812 & 32770 & 89800 \\
\hline 1.5 & 79250 & 70476 & 83734 & 23520 & 83735 \\
\hline 2.0 & 79250 & 67095 & 76136 & 18128 & 76159 \\
\hline 2.5 & 79250 & 63762 & 69572 & 14575 & 69630 \\
\hline 3.0 & 79250 & 60551 & 64513 & 12377 & 64640 \\
\hline
\end{tabular}

The final imperfection model that was investigated was the hyperbolic cylinder, which was examined to investigate its potential to support higher buckling loads. Figure 10 demonstrates that hyperbolic imperfections can have a higher buckling load than a perfect cylinder. For a 1 inch imperfection, the buckling load increases to 89,800 lb (see Table 1). The hyperbolic results also show how closely the buckling loads overlap the hourglass load values. Because the buckling load of the hyperbolic shell is greater than that of the perfect cylinder, a SBKF greater than 1.0 is possible.

A closer investigation of hyperbolic imperfections was performed by refining the imperfection increments to determine the peak buckling load. The hyperbolic equation used to trace the shell imperfections varied the inward apex by 1/8 inch increments from 0 inches to 1-1/2 inches. The buckling loads at these increments are plotted in Fig. 11; the numerical values are given in Table 2. The buckling load increases to a maximum of $90,250 \mathrm{lb}$ at 3/4 inches of imperfection. The buckling load then gradually decreases as the degree of imperfection increases, and eventually decreases below the buckling load of the perfect cylinder. 


\section{Load vs Imperfection}

$\longleftrightarrow$ HYPERBOLIC

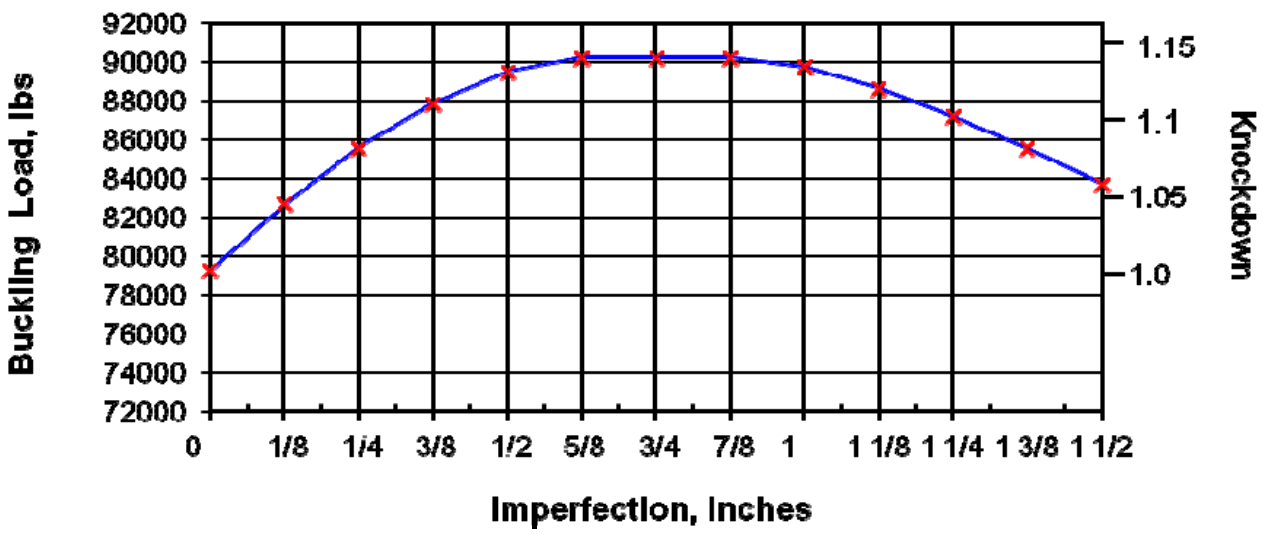

Figure 11. Load versus imperfection results for hyperbolic cylindrical shell.

Table 2. Load versus imperfection results used in Fig. 11.

\begin{tabular}{|c|c|}
\hline $\begin{array}{c}\text { Imperfection, } \\
\text { inch }\end{array}$ & $\begin{array}{c}\text { Buckling load, } \\
\text { lbs }\end{array}$ \\
\hline 0 & 79250 \\
\hline $1 / 8$ & 82705 \\
\hline $1 / 4$ & 85613 \\
\hline $1 / 2$ & 87887 \\
\hline $5 / 8$ & 89526 \\
\hline $3 / 4$ & 90250 \\
\hline $7 / 8$ & 90242 \\
\hline 1 & 90234 \\
\hline $1-1 / 8$ & 89800 \\
\hline $1-1 / 4$ & 88658 \\
\hline $1-1 / 2$ & 87216 \\
\hline $1-3 / 8$ & 85551 \\
\hline $1-1 / 2$ & 83735 \\
\hline
\end{tabular}

\section{Conclusion}

The significant outcome from this investigation is an increased understanding of the effect that manufactured imperfections have on the ability of a cylindrical shell to support axial loads based on the type and degree of imperfection. Skewed, wavy, hourglass, and hyperbolic shapes were investigated and compared to a baseline perfect cylinder result. It was shown that the buckling load for the skewed cylinder continually decreases linearly as the degree of imperfection increases. The wavy imperfection caused the greatest reduction in shell buckling load for a given degree of imperfection.

The opposite behavior was observed in the hourglass imperfection which had a larger buckling load than the perfect cylinder model at the onset of imperfection. This same advantage is observed in modern civil engineering structures (Fig. 12) that use hyperbolic curves to define their shape. These structures have superior buckling stability achieved by inwardly curving their side walls. This geometry was investigated by replacing the hourglass 
imperfection FEM with a hyperbolic side wall cylinder and performing a buckling analysis. The result reveals that this shape is capable of supporting a greater load than the perfect straight wall cylinder. Another advantage in using hyperbolic curves to define structures is that they form a double ruled surface which can be constructed with straight members. This quality makes construction easier and less costly than a circular side wall. The advantages that the hyperbolic side wall offers may diminish when bending moments are introduced on the structure or when manufactured imperfections are too severe.

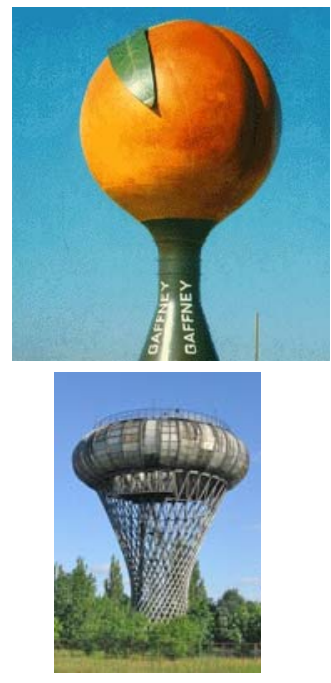

a) Elevated water tanks

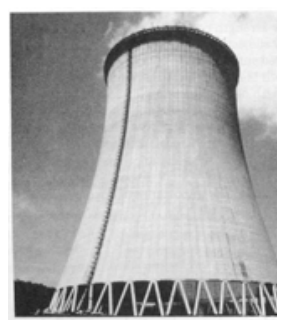

b) Cooling tower

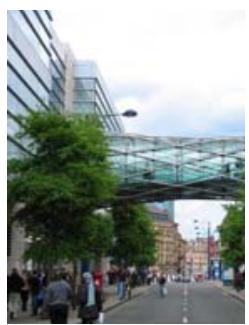

c) Pedestrian bridge

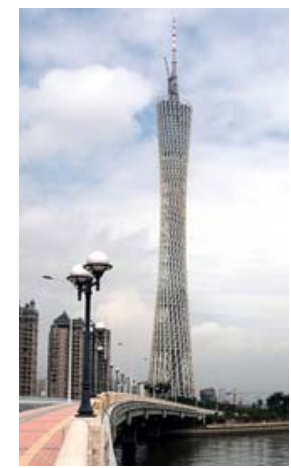

d) Observation Tower

Figure 12. Hyperbolic structures.

\section{References}

${ }^{1}$ Bruhn, E.F., Analysis and Design of Flight Vehicle Structures, Chapter 8, S. R. Jacobs \& Associates, Purdue University, IN, 1973.

${ }^{2}$ Calladine, C. R., "Understanding Imperfection-Sensitivity in the Buckling of Thin-Walled Shells," Thin-Walled Structures, Vol. 23, 1995, pp. 215-235.

${ }^{3}$ Weingarten, V. I., Seide, P. and Peterson, J. P., "Buckling of Thin Walled Circular Cylinders," NASA SP-8007, 1968.

${ }^{4}$ Edlund, B., "Buckling of Metallic Shells: buckling and Postbuckling Behavior of Isotropic Shells, Especially Cylinders," Structural Control and Health Monitoring, 2007, Vol. 14, pp 693-713.

${ }^{5}$ Hornung, U. and Saal, H. "Buckling Loads of Tank Shells with Imperfections," International Journal of Non-Linear Mechanics, Vol. 37, 2002, pp. 605-621.

${ }^{6}$ Teng, J.G. and Rotter, J.M. Buckling of Thin Metal Shells, Spon Press, London, England, 2004, p. 64.

${ }^{7}$ Arbocz, J., Hol J., "Collapse of Axially Compressed Cylindrical Shells with Random Imperfections," Thin-Walled Structures, Vol. 23, 1995, pp. 131-158.

${ }^{8}$ NASA, “SLS Launch Vehicle specifications take shape as development continues,” URL: http://www.nasaspaceflight.com/2012/03/sls-specifications-take-shape-development-continues/ [cited 26 March 2012]. 